\title{
Facteurs liés à l'observance du traitement Antirétroviral (TARV) à l'Unité de soins et d'accompagnements et de Conseils (USAC) de Koulikoro.
}

\section{Compliance related factors of Antiretroviral therapy (ART) at Care unit and accompaniments and Consulting (USAC) Koulikoro.}

\author{
Coulibaly $Y^{1,3}$, Diarra NM 3 , Sangho $F{ }^{1,3}$, Bougoudogo $F$ 2,3, Diallo $D 2,3$
}

1 Direction de la pharmacie et du Médicament, Ministère de la Santé,

2 Institut National de Recherche en santé publique, Ministère de la santé,

3 Faculté de Pharmacie, Université des Sciences, des Techniques et des Technologies de Bamako,

\section{Résumé}

L'objectif était de tester une méthode simple adaptée à un contexte de ressources limitées pour mesurer l'observance et identifier les motifs de non observance rencontrés dans le suivi des traitements antirétroviraux (ARV) en milieu décentralisé au Mali.

II s'agissait d'une étude transversale de juin 2006 à décembre 2008 chez des patients $\mathrm{VIH}$ suivis en consultation dans l'Unité de Soins et d'Accompagnements et de Conseils (USAC) de Koulikoro . C'est ainsi que l'observance pour les ARV a été mesurée. Les patients ont été considérés comme observants si d'après le nombre de comprimés restants ils n'ont sauté aucune prise de leur traitement.

L'étude a porté sur 250 patients. La tranche d'âge 30 à 34 ans était la plus représentée dans la série avec $17,6 \%$. La majorité des patients était de sexe féminin soit $75,1 \%$. La majorité de patients résidait dans la commune urbaine de Koulikoro soit 74,4\%. Le schéma 2 Inhibiteurs Nucléosidiques de la Transcriptase Inverse (INTI) + 1Inhibiteur Non Nucleosidique de la Transcriptase Inverse (INNTI) était le plus prescrit soit $91,6 \%$. Tous les patients étaient sous traitement ARV, avec $78 \%$ sous Triomune (Lamivudine + Stavudine + Nevirapine en combinaison fixe). Les patients qui ont fait un abandon (ou refus) de prendre le médicament étaient majoritaires avec $27,4 \%$. L'observance était de 75,2\%. L'indice global d'observance donne 188 patients $(75,2 \%)$ comme observants et 62 patients $(24,8 \%)$ comme non observants. L'observance thérapeutique dans l'étude est relativement bonne $(75,2 \%$ des patients). Cette étude montre que la mesure de l'observance est possible et faisable en utilisant le comptage des comprimés des patients en milieu décentralisé. Les causes d'inobservances étaient dominées par le refus $(27,4 \%)$; l'oubli $(24,2 \%)$; le voyage $(16,1 \%)$; les effets indésirables des antirétroviraux de type allergique $(12,9 \%)$. Notre étude a montré que $24,8 \%$ des patients ont été non observants en raison du refus, de l'oubli, du voyage et des effets indésirables. Les prestataires ou agents de santé avaient eu plusieurs astuces pour améliorer l'observance des patients telles que relier la prise du traitement à une activité quotidienne ou avant le petit déjeuner. Les associations des PVVIH œuvrent auprès des agents de santé afin de faire accepter aux nouveaux inclus leur statut et surtout le traitement ARV. Mots clés : VIH, Observance, ARV, Koulikoro.

\section{Summary}

Objective: The objective was to test a simple method adapted to the context of resources, limited area to measure adherence, and identify the reasons for noncompliance encountered in the monitoring of antiretroviral therapy (ART) in rural decentralized Mali.

Method: This cross-sectional study in HIV patients followed in the outpatient USAC Koulikoro. Adherence to ART was measured by interviewing patients on regular renewal of the order. Patients were considered adherent if they reported never having failed one or more drug intake and never interrupted treatment.

Results: The study focused on 250 patients. The age group 30 to 34 years was most represented in the series with $17.6 \%$. The majority of patients were female is $75.1 \%$. The majority of patients resided in the urban commune of Koulikoro is $74.4 \%$. Figure 2 NRTIs NNRTI was the one prescribed in our patients is $91.6 \%$. All patients were on ARV treatment, with $78 \%$ under Triomune (Lamivudine + Stavudine + Nevirapine in combination fixed). Patients who have a waiver (or refusal) to take the medication were $27.4 \%$ majority. Compliance was $75.2 \%$ of the overall index of compliance gives 188 patients $(75.2 \%)$ and observant and 62 patients (24.8\%) as non-compliant. Adherence in this study is relatively good $(75.2 \%$ of patients). This study shows that the extent of compliance and feasible using the interview patients decentralized environment. The causes of inobservances were dominated by the refusal $(27.4 \%)$ forgetfulness $(24.2 \%)$ travel $(16.1 \%)$, adverse effects of antiretroviral-type allergy (12.9\%).

Conclusion: In routine clinical practice, its use will assess patient compliance and improve decentralized environment in Mali.

Keywords: HIV, Adherence, ARV, Koulikoro

\section{Introduction}

Le SIDA (Syndrome de l'Immun déficience Acquise) a tué plus de 25 millions de personnes depuis sa première description en 1981, ce qui en fait l'une des épidémies les plus dévastatrices. Au Mali la prévalence est de $1,3 \%$ et de $1,4 \%$ à Koulikoro qui est la $2^{\text {ème }}$ région administrative.

Le coût élevé des thérapies rendait leur accessibilité difficile aux malades dans les pays à ressources limitées. 
La prise en charge des Personnes Vivants avec le VIH (PVVIH) a commencé en 2001 avec l'Initiative Malienne d'Accès aux Antirétroviraux (IMAARV). De la subvention, les ARV sont devenus gratuits en 2005. Cette gratuité augmente la disponibilité. En Mars 2005, fut signé le décret instaurant la gratuité des soins, des ARV, des médicaments pour les infections opportunistes (IO) et du suivi biologique permettant l'accès de la prise en charge aux plus démunis. L'évaluation de l'Initiative Malienne d'Accès aux Antirétroviraux, réalisée en 2005 a, entre autres, montré une nette amélioration de l'accessibilité aux médicaments antirétroviraux depuis l'annonce de la gratuité. Pour optimiser la disponibilité et la prise en charge des PVVIH, l'Unité de Soins d'Accompagnement et de Conseils (USAC) a été mise en place et rendue fonctionnelle le 17 juillet 2005 grâce à un partenariat public-privé-communautaire. L'USAC a été un projet public de site intégré de prise en charge du VIH au soin d'un CSRéf. Des efforts ont été fournis par le gouvernement et ses partenaires pour assurer la disponibilité des ARV aux patients. II était donc nécessaire de vérifier l'observance du traitement ARV au niveau des patients.

L'observance dans le traitement de l'infection par le VIH est un enjeu majeur dans le monde entier et en Afrique en particulier. L'observance dans le cadre de multi thérapies complexes, contraignantes, avec un risque d'effets secondaires important et durant plusieurs années est un défi. Néanmoins, elle est absolument nécessaire si on veut préserver l'efficacité des médicaments actuels et à venir [1-3]. La plupart des études montrent que 40 à $60 \%$ des patients ont un niveau d'observance inférieur à $90 \%[4,5]$. A notre connaissance, II n'existe pas de données sur l'observance des patients en milieu décentralisé à Koulikoro depuis la gratuité des antirétroviraux (ARV). Partant de ce constat, il est primordial de déterminer l'influence de ces facteurs sur l'observance afin d'élaborer des stratégies pour amener les patients à une meilleure observance thérapeutique. Le but de notre étude était de déterminer les facteurs associés à l'observance du TARV à l'Unité de Soins et d'Accompagnements et de Conseils (USAC) de Koulikoro.

\section{Matériel et Méthode}

L'étude s'est déroulée à l'Unité de Soins et d'Accompagnements et de Conseils des personnes vivants avec le VIH de Koulikoro. L'USAC a été intégrée au Centre de Santé de Référence (CSRéf) de Koulikoro. II s'agissait d'une étude transversale qui s'est déroulée de janvier 2006 à décembre 2008. La population d'étude était constituée par les PVVIH sous traitement antirétroviral suivis dans l'USAC de Koulikoro. Les patients ayant au moins 6 mois de traitement ARV, et ayant consenti à participer à l'étude ont été inclus. L'échantillonage a concerné 250 sujets consentants et informés. Les données ont été recueillies à partir du comptage des comprimés restant lors du renouvellement de l'ordonnance, et en fonction de la date de sa dernière visite, il détermine si le patient a sauté des prises ou pas à l'aide d'un questionnaire et d'un examen documentaire (dossier patient) Nos critères d'inobservance étaient les suivants : tout patient ayant pris ses médicaments avec un décalage horaire de plus d'une heure, tout patient ayant omis une ou plusieurs prises médicamenteuses, tout patient ayant interrompu le traitement. Nous avons apprécié également le rythme de renouvellement des ordonnances à partir du logiciel de la pharmacie (ESOPE) et des dossiers des malades. Dans le souci de préserver les considérations éthiques, une autorisation préalable des autorités du centre de Santé de Référence de Koulikoro, en l'occurrence la direction et les responsables du service de I'USAC a été obtenue. Aussi, le consentement éclairé verbal de tous les patients a été obtenu. Les données ont été saisies et analysées grâce aux logiciels SPSS.

\section{Résultats}

Nous avons enquêté auprès de 250 patients sous traitement ARV suivi à I'USAC de Koulikoro de janvier 2006 à décembre 2008. Notre étude s'est portée sur $61,58 \%$ de l'effectif (250/406). Les patients de sexe féminin étaient plus nombreux avec $75,1 \%$. La majorité des patients résidaient dans la commune urbaine de Koulikoro soit $74,4 \%$. Les patients étaient de sexe féminin dans $75,2 \%$ des cas. Sur l'ensemble de l'échantillon, la tranche d'âge la plus active ( 25 - 39 ans) constitue $58 \%$ des cas. La tranche 30 à 34 ans était la plus représentée avec $17,6 \%$ des cas. Dans notre étude $91,6 \%$ des patients ont commencé leur traitement avec 2 INTI + 1INNTI. Le schéma Stavudine(D4T)+ lamivudine (3TC)+ nevirapine (NVP) était le plus prescrit dans $78 \%$ des cas. Sur les 250 patients, $75,2 \%$ des patients étaient observants selon nos critères (Tableau I). Chez les patients inobservants $70,9 \%$ ont fait un arrêt, suivis des patients qui ont fait deux arrêts avec $24,2 \%$, enfin ceux qui ont fait trois arrêts $4,8 \%$ (Tableau II). Les causes d'inobservances étaient dominées par l'abandon (refus) $(27,4 \%$ ) ; l'oubli $(24,2 \%)$; le voyage $(16,1 \%)$; les effets indésirables des antirétroviraux $(12,9 \%)$, le social des patients $(9,7 \%)$, le manque de moyen de transport $(8,1 \%)$ et les occupations de la famille $(1,6 \%)$ (Tableau III). Les effets indésirables étaient dominés par les troubles digestifs ( nausées, vomissements et diarrhée) suivis des intolérances neurologiques ( céphalée, cauchemars, insomnie et neuropathie périphérique) et les allergies cutanées. Les patients ayant parcouru moins de $50 \mathrm{~km}$ représentent $95,2 \%$, ceux ayant parcouru plus de $50 \mathrm{~km}$ représentent $4,8 \%$.

\section{Discussion}

Alors qu'il n'existe pas de méthode de référence pour une évaluation quantitative de l'observance, le choix en Afrique subsaharienne des méthodes est relativement limité en raison de l'absence des moyens techniques (les dosages des ARV ou l'usage de piluliers électroniques ne sont pas envisageables localement) et du niveau d'éducation des patients concernés (auto-questionnaires non adéquats dans une population à fort pourcentage d'illettrés) $[4,5]$. Notre étude a connu les mêmes limites méthodologiques que les autres travaux mesurant 
l'observance par les questionnaires. Ces déclarations peuvent être sujettes à des biais de mémorisation ou de conformisme social [4]. L'approche transversale comporte également des limites ne permettant pas d'observer la dynamique de l'observance et des facteurs qui la rythment. La force de notre étude réside donc dans la nécessité d'apporter un soulagement au malade, de permettre au pharmacien dispensateur de pouvoir être à mesure de prodiguer les conseils utiles aux malades pour une bonne observance et une adéquation dans la vie du malade. Pendant la période d'étude, il y a eu rupture de stock de la névirapine (NVP) $200 \mathrm{mg}$. Mais cela n'a pas entraîné un arrêt de traitement chez les patients qui étaient sous cette molécule. Elle a été remplacée par l'efavirenz (EFV) $600 \mathrm{mg}$ durant cette période de rupture.

Les données sociodémographiques sont comparables à celles de la littérature [5-9]. Dans notre étude le schéma 2 INTI + 1INNTI a été le plus représenté soit $91,60 \%$. Ces résultats sont en accord avec les recommandations nationales de prise en charge des personnes Vivants avec le VIH du Mali. Les changements de molécules dans les prescriptions étaient dus essentiellement à des effets secondaires suivis de rupture de stock. Dans notre étude l'association lamivudine (3TC)-stavudine (D4T)-névirapine(NVP) (triomune) a été la plus prescrite soit $78 \%$ correspondant au schéma 2 INTI + 1INNTI conformément aux recommandations du Mali. Ce constat permettra dans les jours à venir de planifier les besoins et l'approvisionnement en ARV. Les premiers résultats montrent que l'observance des schémas thérapeutiques antirétroviraux simplifiés, en particulier ceux des médicaments en associations fixes, est très élevée (environ 81,20\%), proche de celui de Oumar et al en 2007 [9]. Dans notre étude, nous avons noté une prédominance féminine avec $72,4 \%$. Ce résultat est supérieur à celui de Oumar et al en 2007 à Bamako [9] avec $63,2 \%$ au Mali. Dans notre étude la moyenne d'âge est égale à 38,5 ans avec des extrêmes de 12 à 65 ans. La tranche d'âge (20-44 ans) était majoritaire avec $68 \%$. Ce résultat est supérieur à celui Oumar et al en 2007 [9] qui avait trouvé une classe d'âge de (30-39 ans) 63,80\%. Dans notre étude, nous avons noté une prédominance des femmes au foyer $(50,40 \%)$, suivent ensuite les cultivateurs $(13,60 \%)$, les étudiants et les commerçants avec respectivement $(8,80 \%$ et $8,40 \%)$. Des efforts doivent être fournis pour la sensibilisation de la population à faire le dépistage avant le mariage. Dans notre étude, en plus de Koulikoro les localités de Banamba, Bamako, Kangaba, Nara et Bougouni ont été représentées. Ce qui démontre l'ampleur de la pandémie et une nécessité absolue de continuer avec une décentralisation de la prise en charge des $\mathrm{PVVIH}$, les Koulikorois ont été les plus représentés soit $74,4 \%$. Ce constat a été aussi observé par Oumar et al en 2007[9] à Bamako. II est à signaler aussi que la distance et le manque de moyens financier pour le transport posent d'énormes problèmes aux patients pour la bonne observance au traitement. En dépit de ces contraintes, seulement $24,8 \%$ des patients sont inobservants. Le taux global d'observance était de $75,2 \%$ est inférieur à ceux du Sénégal [10], à ceux du Djibouti [11] et de l'Ethiopie [12], mais supérieur à ceux du Burkina Faso [13] et du Mali $[9,14]$. Ce taux d'observance des patients est plus élevé comparé aux autres études africaines $[9,13,14,15]$. Cette situation peut s'expliquer par la différence des approches méthodologiques. Le mode de vie joue un rôle très important dans la persévérance du traitement des malades. L'observance de nos patients a été favorisée par plusieurs facteurs : (la compréhension du traitement, l'assiduité dans la prise des molécules, l'assistance des patients par leurs proches, les schémas simplifiés par les formes associées).

La proportion de sujets inobservants dans notre étude était de $24,8 \%$.est sensiblement du même ordre que celui de Maly et al. Au Cameroun [7]. Parmi ces facteurs nous avons marqué les prises manquées, la non adhésion, les effets secondaires et l'abandon. La majorité des prises manquées étaient dues plus fréquemment à l'oubli. L'accès difficile pour certains de nos patients venant des zones reculées de Koulikoro était aussi une source d'inobservance. Les antirétroviraux ne sont pas des médicaments anodins. Ils présentent tous une certaine toxicité pour l'organisme, responsable d'effets secondaires ou indésirables plus ou moins marqués à court, à moyen et à long terme. Ces effets secondaires sont variés, bénins ou graves et différents selon le moment de leur apparition et de la classe ou du type de molécule utilisée. Ils peuvent inciter à l'arrêt du traitement par certains patients. Ainsi au cours de notre étude nous avons pu observer que les effets secondaires constituaient un obstacle pour $12,9 \%$ de nos patients. Les patients ayant ressenti des effets secondaires ont été moins observants. Parmi les manifestations indésirables, celles touchant le système digestif: les nausées, vomissements, diarrhées étaient majoritaires suivies des intolérances neurologiques : les céphalées, les cauchemars, l'insomnie, puis celles touchant le système nerveux : neuropathie périphérique. Laurent et al. ont rapporté des résultats similaires au Sénégal en 2002 [10]. Cependant l'inobservance était liée à la survenue des certains effets indésirables comme décrit dans d'autres études [11,12,14-17].

\section{CONCLUSION}

Le taux d'observance à l'USAC de Koulikoro $(75,2 \%)$ est acceptable. Nos résultats révèlent que l'évaluation de l'observance au cours du traitement doit être régulière, mais également indispensable pour la prise des traitements. Nous recommandons la formation du personnel et la mise en place d'un programme d'éducation thérapeutique pour les PVVIH. L'observance constitue un défi du fait de la complexité et de la durée du traitement

\section{Références}

1. Knobel H, Carmona A, Grau S et al. Adherence and effectiveness of highly active antiretroviral therapy. Arch Intern Med $1998 ; 158: 1953$. 
2. Cohen OJ, Fauci AS. Transmission of multidrugresistant human immunodeficiency virus. The wakeup call. N Engl J Med 1998 ; 339 : 341-3.

3.Wainberg MA, Friedland GH. Public health implications of antiretroviral therapy and HIV drug resistance. JAMA $1998 ; 279$ : 1977-83.

4. Marcyl O, Bakala N. Suivi de l'observance et éducation du patient sous ARV en Afrique: l'expérience du CTA de pointe-Noire. Développement et santé 2004, 172.

5. Andréo $C$, Bouhnik $A D$, Soletti $J$ et al. La non observance des patients infectés par le $\mathrm{VIH}$, soutenus par une association communautaire. Santé publique $2001 ; 13: 249-62$.

6. Laurent C, Meilo H, Guiard-Schmid JB et al. Antiretroviral therapy in public and private routine health care clinics in Cameroon : Lessons from the Douala Antiretroviral (DARVIR) Initiative. Clinical Infectious Diseases 2005 ; 41 : 108-11.

7. Mahy S, Duong M, Huraux JM et al. [Measure of effectiveness and adherence to antiretroviral therapy in HIV-infected patients in Cameroon]. Med Mal Infect $2011 ; 41: 176-80$.

8. Laurent C, Gueye FNN, Ndour CT et al. Long-Term benefits of highly active Antiretroviral therapy in Senegalese HIV-1-infected Adults. AIDS 2005 ; 38, $\mathrm{N}^{\circ} 1: 14-17$.

9.Oumar AA, Dao S, Diamoutene A et al. Les Facteurs associés à l'observance du traitement antirétroviral à l'hôpital du Point G. Mali Med 2007, 22 (1) :25-29.

10. Laurent C, Diakhate N, Gueye FNN, et al. The Senegalese government's highly active antiretroviral therapy initiative: an 18-monh follow-up study. AIDS $2002 ; 16(10): 1363-1370$

11. Ahmed AA, Katlama C, Ghosn J et al. Evaluation de l'observance au traitement antirétroviral au sein d'une cohorte de 200 patients à Djibouti (2005). Eastern Mediterranean Health Journal $2005 ; 13: 1-13$.

12. Beyene KA, Gedif T, Gebre-Mariam T et al. Highly active antiretroviral therapy adherence and its determinants in selected hospitals from south and central Ethiopia. Pharmacoepidemiol Drug Saf 2009 ; $18: 1007-15$.

13.Ouedrago M., Bambara M., Zougba AZ, et al. Intérêts et contraintes des traitements antirétroviraux dans un pays en développement. Méd Afr Noire 2001 ; (7) :321-24.

14.Oumar AA, Demebele JP, Diarra $S$ et al. Les facteurs associés à l'inobservance du traitement antirétroviral dans un pays à ressources limitées : le cas du Mali. Revue Tunisienne d'Infectiologie. Octobre 2012, Vol.6, Nº : 203 - 206

15. Mouala C, Kaba-Mebri J, Wata JB et al. Factors associated with adherence to therapy among HIVinfected patients in Bangui. Sante 2006 ; 16 : 119-30.

16. Bisson GP, Gross R, Bellamy $S$ et al. Pharmacy refill adherence compared with CD4 count changes for monitoring HIV-infected adults on antiretroviral therapy. PLoS Med $2008 ; 5:$ e109.

17. Gherissi A, Tinsa F. Étude des déterminants de l'observance de la trithérapie auprès des personnes vivant avec le VIH en Tunisie, 2008. Rev Tun Infectiol $2010 ; 4: 66-73$

Tableau I: Comparaison de la méthode de mesure de l'observance de notre étude par rapport aux autres études africaines

\begin{tabular}{|c|c|c|c|c|c|}
\hline Etude & Année & Pays & Méthode & Taille & $\begin{array}{l}\text { Taux } \\
\text { observance }\end{array}$ \\
\hline Oumar et al & 2007 & Mali & Dénombrement des comprimés & 345 & 41,8 \\
\hline Oumar et al & 2012 & Mali & Interview & 270 & 56,3 \\
\hline Ahmed et al & 2005 & Djibouti & Dénombrement des comprimés & 86 & 77 \\
\hline Moula et al & 2006 & RCA & $\begin{array}{l}\text { Auto-questionnaires et dénombrement } \\
\text { des comprimés }\end{array}$ & 89 & $69-78$ \\
\hline Beyene et al & 2009 & Ethiopie & $\begin{array}{l}\text { Interview et dénombrement des } \\
\text { comprimés }\end{array}$ & 422 & $93,1-88,1$ \\
\hline Laurent et al & 2002 & Sénégal & Interview & 58 & 87,9 \\
\hline Ouedraogo et al & 2001 & Burkina & Interview & 34 & 50 \\
\hline Notre Etude & 2013 & Koulikoro & $\begin{array}{l}\text { Dénombrement des comprimés et } \\
\text { Interview }\end{array}$ & 250 & 75,2 \\
\hline
\end{tabular}


Tableau II : Répartition des patients inobservants en fonction du nombre d'arrêts du traitement ARV.

\begin{tabular}{lcccc}
\hline Nombre & \multicolumn{3}{c}{ Sexe } & \\
d'arrêt & 6 & 38 & 44 & Pourcentage \\
\cline { 2 - 3 } & Masculin & Féminin & Total & 70,97 \\
\hline Un arrêt & 3 & 12 & 15 & 24,19 \\
Deux arrêts & 1 & 2 & 3 & 4,84 \\
Trois arrêts & 10 & 52 & 62 & 100 \\
\hline Total & & &
\end{tabular}

Tableau III : Répartition des patients inobservants selon les causes d'inobservance.

\begin{tabular}{|c|c|c|c|c|c|c|}
\hline & & & \multirow{4}{*}{ Total } & \multirow{4}{*}{$\%$} & & \\
\hline & \multicolumn{2}{|c|}{ Sexe } & & & \multicolumn{2}{|c|}{ Age moyen (année) } \\
\hline & \multirow[t]{2}{*}{ Masculin } & \multirow[t]{2}{*}{ Féminin } & & & & \\
\hline & & & & & Masculin & Féminin \\
\hline Oubli & 1 & 14 & 15 & 24,2 & 35 & 31,57 \\
\hline Effets indésirables & 2 & 6 & 8 & 12,9 & 33,5 & 32,83 \\
\hline Refus & 3 & 14 & 17 & 27,4 & 41,33 & 30 \\
\hline Social & 0 & 6 & 6 & 9,7 & 0 & 28,17 \\
\hline Voyage & 2 & 8 & 10 & 16,1 & 55,50 & 32,38 \\
\hline Occupation & 0 & 1 & 1 & 1,6 & 0 & 26 \\
\hline Moyen de Transport & 2 & 3 & 5 & 8,07 & 41,50 & 39,33 \\
\hline Total & 10 & 52 & 62 & 100 & 1 & 1 \\
\hline
\end{tabular}

